Año 13.

Académica sin Frontera

Núm. 34 Núm. Especial. Tema COVID 19.

ISSN: 2007-8870

https://revistainvestigacionacademicasinfrontera.unison.mx/index.php/RDIASF

Recibido el 20 de agosto de 2020. Dictaminado mediante arbitraje favorablemente 7 de diciembre de 2020.

\title{
Proceso de enseñanza - aprendizaje durante COVID-19
}

\author{
Dra. María Lizett Zolano Sánchez \\ maria.zolano@unison.mx \\ https://orcid.org/0000-0001-7526-2038 \\ Universidad de Sonora, Unidad Norte \\ Dra. María Julia León Bazán \\ julia.leon@unison.mx \\ https://orcid.org/0000-0002-7405-6141 \\ Universidad de Sonora, Unidad Centro
}

\begin{abstract}
Resumen
Este trabajo de investigación se realizó bajo un enfoque metodológico exploratorio, no experimental y descriptivo, tomando como referencia a 75 docentes de la División de Ciencias Administrativas, Sociales y Agropecuarias, Unidad Norte de la Universidad de Sonora adscritos durante el semestre 2020-1. El objetivo es identificar, desde la perspectiva de los docentes universitarios, los principales retos a los que se enfrentan en el proceso de enseñanza - aprendizaje durante la contingencia causada por el COVID-19. Los resultados obtenidos muestran que los docentes no utilizan las plataformas virtuales como herramienta de apoyo en el proceso de enseñanza- aprendizaje debido al desconocimiento en el uso, gestión y aprovechamiento de los espacios virtuales dentro de los modelos pedagógicos utilizados. En conclusión, mejorar los procesos de docencia e investigación y trabajar en pedagogías alternativas son los principales desafíos de cara a la nueva normalidad educativa.
\end{abstract}

Palabras Clave: Enseñanza-Aprendizaje, COVID-19, Crisis Sanitaria, Desafío, Docente. 


\title{
Teaching and learning process during COVID-19 English Spanish translator
}

\begin{abstract}
This research work was carried out under an exploratory, non-experimental and descriptive methodological approach, taking as a reference 75 teachers from the Division of Administrative, Social and Agricultural Sciences, North Unit of the University of Sonora assigned during the 2020-1 semester. The objective is to identify, from the perspective of university teachers, the main challenges they face in the teaching-learning process during the contingency caused by COVID-19. The results obtained show the teachers do not use virtual platforms as a support tool in the teaching-learning process due to the lack of knowledge in the use, management and profiting of virtual spaces within the pedagogical models used. In conclusion, improving teaching and research processes and working on alternative pedagogies are the main challenges in the face of the new normal of education.
\end{abstract}

Key words: Teaching-Learning, COVID-19, Health Crisis, Challenge, Teacher.

\section{Introducción}

Desde hace algún tiempo, algunos expertos advertían que la tecnologización de la vida humana requería profesionales óptimamente capacitados en el uso de Tecnologías de la Información y la Comunicación (TIC's), lo cual excluye a todo aquel que no logre adaptarse a las nuevas condiciones. Sugerían, en vistas de ese futuro irreversible, una reconfiguración de los currículos universitarios para que la formación continua de docentes se enfocara a incluir actitudes, habilidades y competencias relacionadas con la tecnología, como un plus a su formación para poder enfrentar con eficiencia los contenidos de las materias de cualquier ámbito de estudio (Barrios, 2013).

A partir del siglo XXI se hizo frecuente el uso de las TIC's como herramientas didácticas del proceso de enseñanza - aprendizaje porque es un medio que contribuye al desarrollo de habilidades y destrezas, a la vez que mejoran el aprendizaje, pero también es cierto que sigue existiendo como un obstáculo para su plena inclusión en las aulas, debido a la formación tecnológica y la actitud del docente y el propio alumno porque aun utilizan con regularidad las presentaciones de diapositivas electrónicas elaboradas en Power Point o Prezi en el desarrollo y contenido de la clase (Navarro, Robles, Leyva y Lugo 2016; Granda, Espinoza y Mayon, 2019, Barráez, 2020). 
Año 13.

Núm. 34 Núm. Especial. Tema COVID 19.

ISSN: 2007-8870

https://revistainvestigacionacademicasinfrontera.unison.mx/index.php/RDIASF

Recibido el 20 de agosto de 2020. Dictaminado mediante arbitraje favorablemente 7 de diciembre de 2020.

En el campo educativo el uso de las TIC’s se están adueñando de un espacio preferencial en comparación con los recursos utilizados de forma tradicional dentro del proceso de enseñanza - aprendizaje, para lo cual es de vital importancia reconocer que las TIC's garantizan un nivel de aprendizaje acorde a los procesos establecidos como parte de las actividades académicas ya es una realidad dentro de las universidades dando como resultado una institución activa, que garantiza el desarrollo integral de los estudiantes y el desarrollo de nuevas formas de aprendizaje porque desarrolla habilidades y destrezas, mejorando los procesos en el aprendizaje de los estudiantes y de los mismos docentes (Lorca, Cuenca, Vázquez-Bernal y Lorca, 2016; Álava, Illescas y Loor, 2017; Alcívar et al. 2019).

A partir de la pandemia provocada por el COVID-19, se ha obligado a docentes y alumnos a transformar su enseñanza de modalidad presencial hacia la denominada "enseñanza remota," un modelo que se ha suscitado desde la emergencia que se esta experimentando con el uso de sesiones sincrónicas en vivo y el uso masivo de plataformas virtuales donde el proceso de enseñanza - aprendizaje se realiza a través de las Tecnologías de la Información y la Comunicación (TIC’s), herramientas didácticas útiles para este caso pero donde los principales obstáculos para su plena inclusión en las aulas están en la formación tecnológica y la actitud del docente (Granda, Espinoza y Mayon, 2019).

Hoy en día, es un reto urgente dentro de la formación del docente universitario como agente clave, adaptarse a las nuevas condiciones de virtualización de manera emergente en el contexto de la crisis y responder al tipo de profesional que exige la sociedad (Suárez, 2020; García, 2020). Sin embargo, a pesar de que la educación mediada por tecnologías lleva varias décadas en la educación formal, también lo es la resistencia evidenciada en algunos docentes a abandonar prácticas tradicionales a favor de las innovaciones que proponen las tecnologías al servicio del aprendizaje y, durante la contingencia provocada por la pandemia de COVID-19, 
continúan en función con el impacto de nuevos estresores tales como la divergencia en la planificación, desarrollo y evaluación de las clases así como también la incidencia de los medios de comunicación y las redes sociales en los procesos de enseñanza - aprendizaje (Villafuerte, Cevallos, y Vidal, 2020).

Para todas las universidades, el COVID-19 ha forzado un cambio enorme y abrupto, pero probablemente inevitable que le lleva a acelerar el uso y la adecuación de la educación en línea, lo que ha evidenciado una problemática ya conocida, como es la desigualdad en el acceso a la tecnología y su aplicación eficaz (Archer y De Gracia, 2020). Suspender las actividades presenciales en las universidades ocasionó que se transfirieran de modo inmediato todas ellas a un formato online, provocando que esta acción, urgente y sobrevenida, sea análoga en experiencia, planificación y desarrollo a las propuestas que están específicamente diseñadas desde su concepción para realizar un proceso de enseñanza-aprendizaje online (Hodges, Moore, Lockee, Trust y Bond, 2020). Basado en lo anterior, se deduce la importancia del presente trabajo de investigación, debido a que el resultado del proyecto ayudará a cumplir con el objetivo de determinar, desde la perspectiva de los docentes universitarios, los principales retos a los que se enfrentan en el proceso de enseñanza - aprendizaje durante la contingencia causada por el COVID-19.

\section{Materiales y Método}

En este documento se presentan los resultados de una investigación de campo bajo un enfoque metodológico de tipo descriptiva y exploratoria y no experimental y su objetivo es identificar, desde la perspectiva de los docentes universitarios, los principales retos a los que se enfrentan en el proceso de enseñanza - aprendizaje durante la contingencia causada por el COVID-19. Se realizó en la División de Ciencias Administrativas, Sociales y Agropecuarias, Unidad Norte de la Universidad de Sonora que cuenta con tres departamentos: 1) Departamento de Ciencias Administrativas y Agropecuarias con 26 docentes, de los cuales 11 son de Tiempo Completo Indeterminados, 12 son Maestros de Asignatura Indeterminados y 3 de Asignatura Determinados; 2) Departamento de Ciencias Económicas y Administrativas; con 19 docentes, de los cuales 5 son de Tiempo Completo Indeterminados, 9 son Maestros de Asignatura Indeterminados y 5 de Asignatura Determinados; 3) Departamento de Ciencias Sociales; con 48 docentes, de los cuales

7 son de Tiempo Completo Indeterminados, 5 son Maestros de Asignatura Indeterminados y 36 


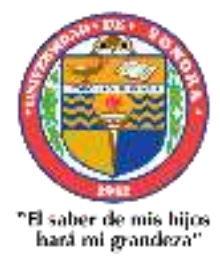

( Julio - Diciembre 2020)

Año 13.

Núm. 34 Núm. Especial. Tema COVID 19.

ISSN: 2007-8870

https://revistainvestigacionacademicasinfrontera.unison.mx/index.php/RDIASF

Recibido el 20 de agosto de 2020. Dictaminado mediante arbitraje favorablemente 7 de diciembre de 2020.

de Asignatura Determinados. Se tomó como muestra a un total de 75 docentes de una población de 93 adscritos en el semestre 2020-1.

Para este estudio se aplicó un cuestionario estandarizado (validado a juicio de expertos y con un coeficiente de confiabilidad Alpha de Cronbach de 0,92 Muy Alta) por (González-Díaz \& Perez, 2015) con 12 items mediante un Google form (González-Díaz et al., 2016). El 30 de abril del 2020, se envió a los correos institucionales de forma masiva y simultánea a los 15 profesores de la muestra elegida, en el correo se incluía una breve invitación a responder de forma voluntaria y, que de aceptar tenían 3 días como margen para dar respuesta a través de la liga al instrumento de la plataforma de Google Forms; esta herramienta permitió capturar, monitorear y organizar las respuestas en tiempo real. Los resultados del Google Forms se exportaron a Google Sheels, para realizar la selección, traducción, transposición y codificación de los datos, y generar las estadísticas descriptivas y las gráficas de los resultados. 


\section{Resultados}

Se presentan a continuación los resultados más relevantes de los avances de la investigación basados en el objetivo central del estudio:

Imagen 1. Experiencia docente en la Universidad de Sonora

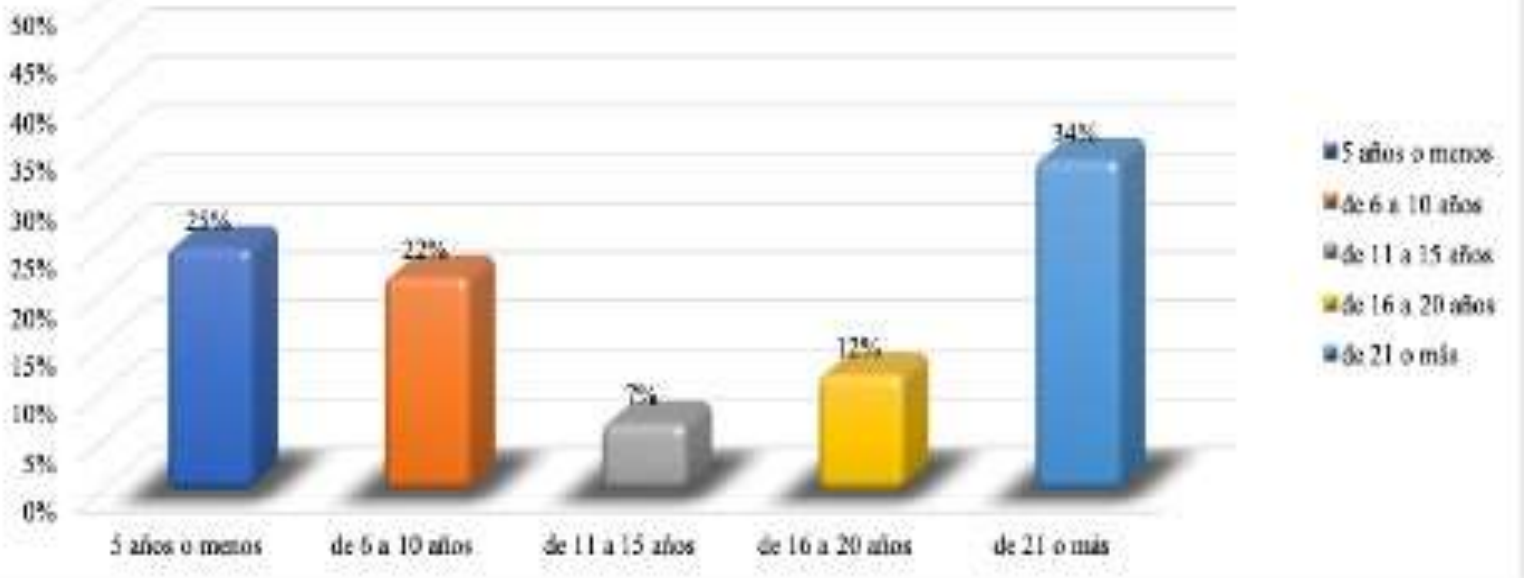

Fuente: Elaboración propia en base a resultados de la investigación.

La imagen 1, muestra que el 34\% de los docentes de la División tiene 21 años o más de experiencia docente dentro de la universidad, el 25\% 5 años o menos, el 22\% de 6 a 10 años, el $12 \%$ tiene de 16 a 20 años y un 7\% de 11 a 15 años de experiencia docente.

Imagen 2. Frecuencia en el uso de las TIC's previo a la pandemia provocada por el COVID-19

$55 \%$
$50 \%$
$45 \%$
$40 \%$
$35 \%$
$30 \%$
$25 \%$
$20 \%$
$15 \%$
$10 \%$
$5 \%$
$0 \%$

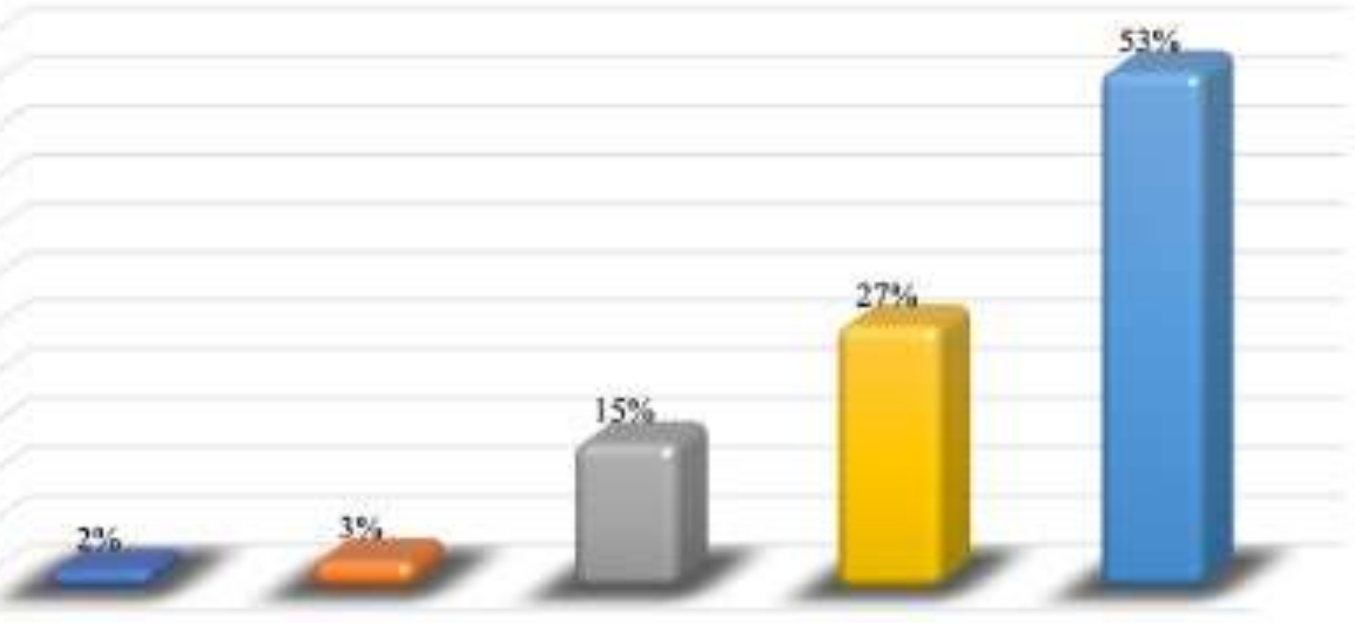
a. Siempre
b. Casi siempre
c. Algunas veces
d. Casi nunca
e. Nunca 
Año 13.

Académica sin Frontera

Núm. 34 Núm. Especial. Tema COVID 19.

ISSN: 2007-8870

https://revistainvestigacionacademicasinfrontera.unison.mx/index.php/RDIASF

Recibido el 20 de agosto de 2020. Dictaminado mediante arbitraje favorablemente 7 de diciembre de 2020.

Fuente: Elaboración propia en base a resultados de la investigación.

La imagen 2, muestra que el 53\% de los docentes de la División nunca ha utilizado las TIC's como instrumento de apoyo en el proceso de enseñanza-aprendizaje aplicado previo a la pandemia provocada por el COVID-19, el 27\% menciona que casi nunca las utiliza, el $15 \%$ algunas veces lo hace, el $3 \%$ casi siempre y el $2 \%$ siempre.

Imagen 3. Principal espacio virtual utilizado

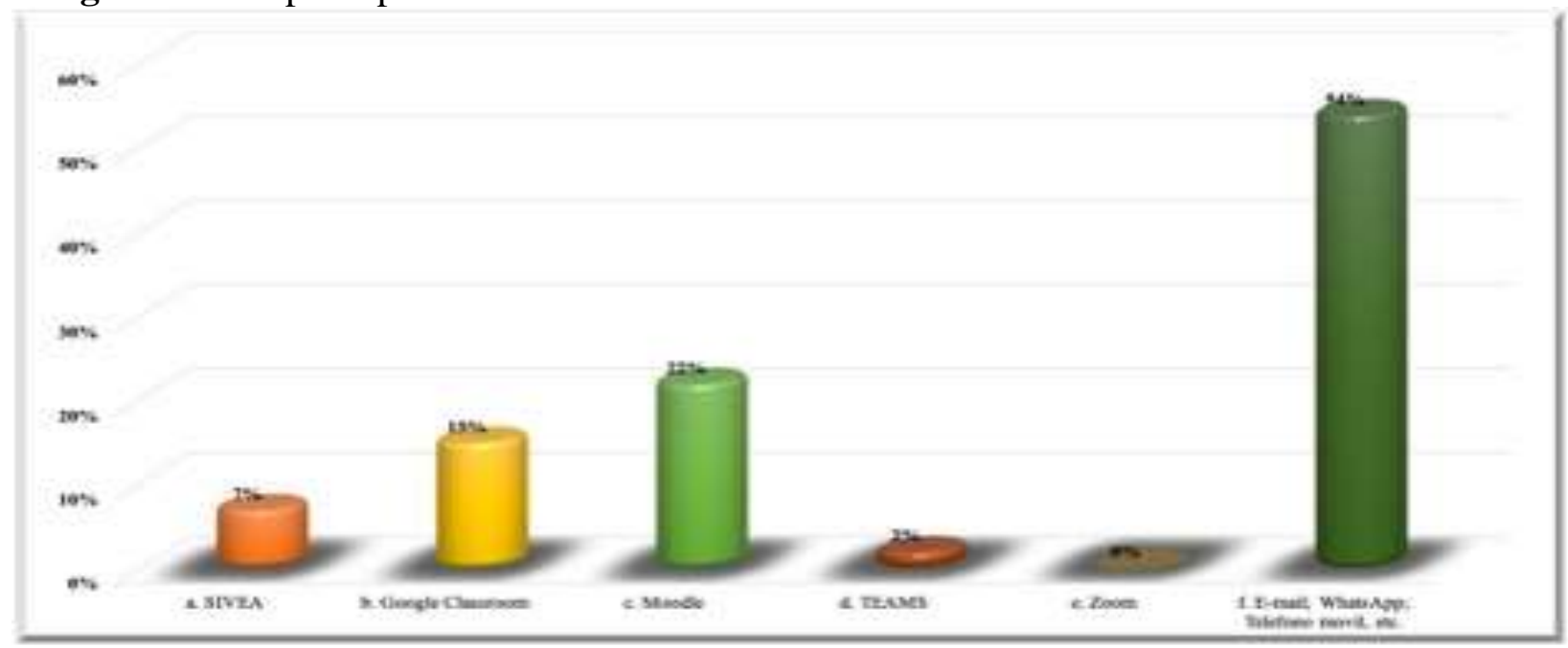

Fuente: Elaboración propia en base a resultados de la investigación.

La imagen 3, muestra que el 54\% de los docentes utilizan el e-mail, WhatsApp, teléfono movil etc., como principal espacio virtual en el proceso de enseñanza-aprendizaje durrante el confinamiento provocado por la pandemia COVID-19, el 22\% utiliza plataforma Moodle, el 15\% el Google Classroom y el 7\% la plataforma institucional SIVEA y el 2\% TEAMS. 


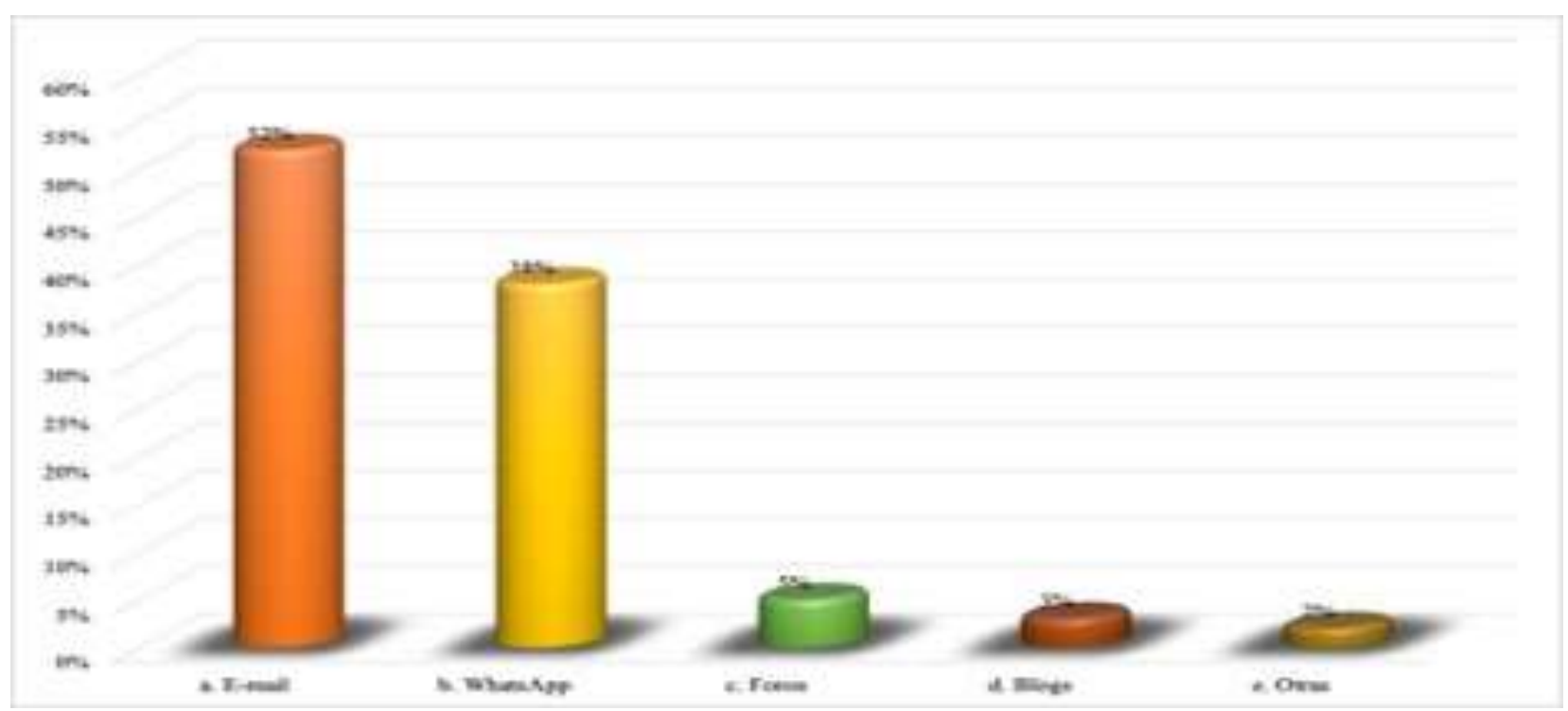

Fuente: Elaboración propia en base a resultados de la investigación.

La imagen 4, muestra que el $52 \%$ de los docentes utilizan el E-mail como medio de comunicación con los alumnos durante el proproceso de enseñanza-aprendizaje durante el confinamiento, el $18 \%$ utiliza el WhatsApp, el $5 \%$ los foros, el $3 \%$ hace uso de blogs y el $2 \%$ otros.

Imagen 5. Principal problema en la implementación de modelos de educación virtual en momentos de confinamiento

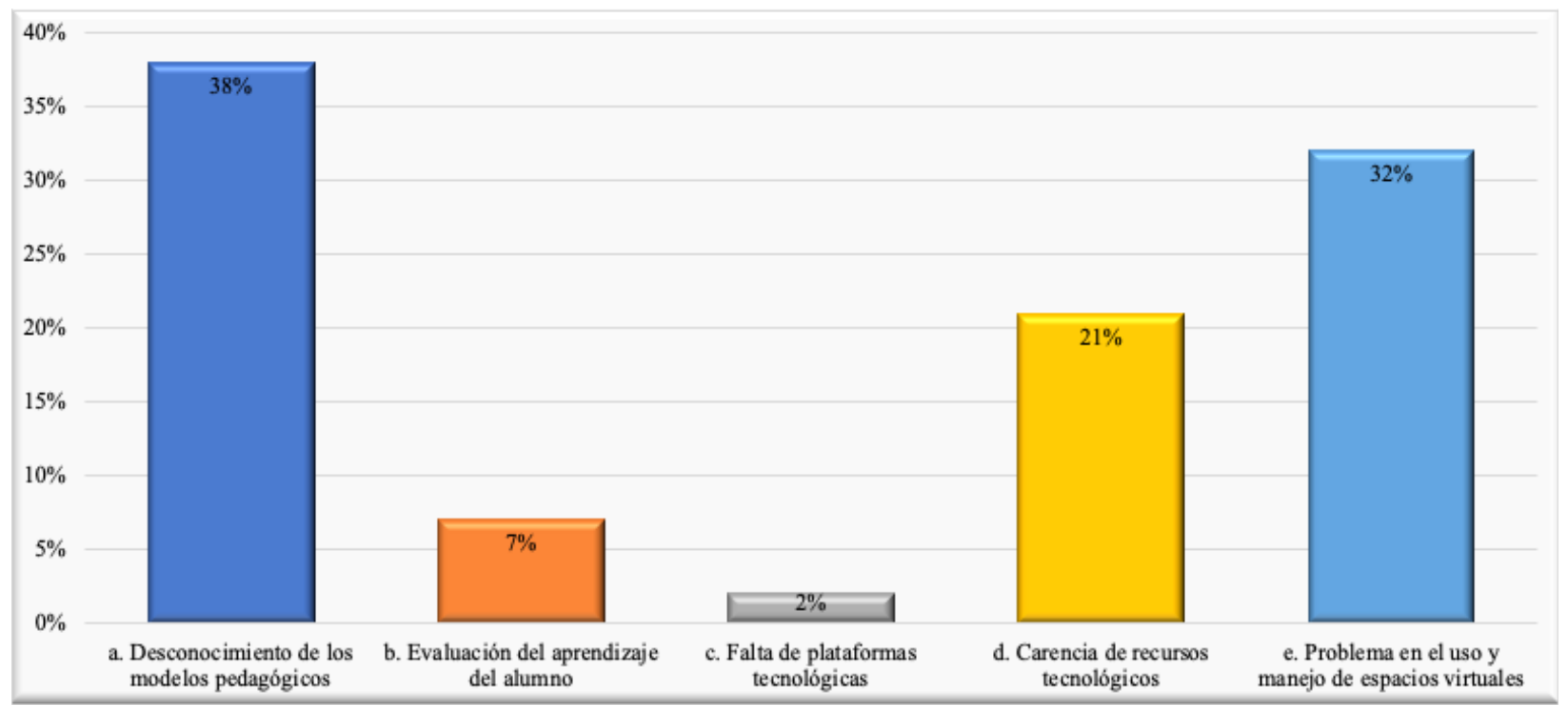

Fuente: Elaboración propia en base a resultados de la investigación. 
Año 13.

Académica sin Frontera

Núm. 34 Núm. Especial. Tema COVID 19.

ISSN: 2007-8870

https://revistainvestigacionacademicasinfrontera.unison.mx/index.php/RDIASF

Recibido el 20 de agosto de 2020. Dictaminado mediante arbitraje favorablemente 7 de diciembre de 2020.

La imagen 5, muestra que desde la perspectiva del $38 \%$ de los docentes de la muestra analizada, el mayor problema encontrado es el desconocimiento de los modelos pedagógicos, seguida con un $32 \%$ que menciona los problemas en el uso y manejo de espacios virtuales, el $21 \%$ la carencia de recursos tecnológicos, el 7\% la evaluación del aprendizaje del alumno y el 2\% la falta de plataformas tecnológicas.

Imagen 6. Interacción con los estudiantes, colegas y directivos de la institución.

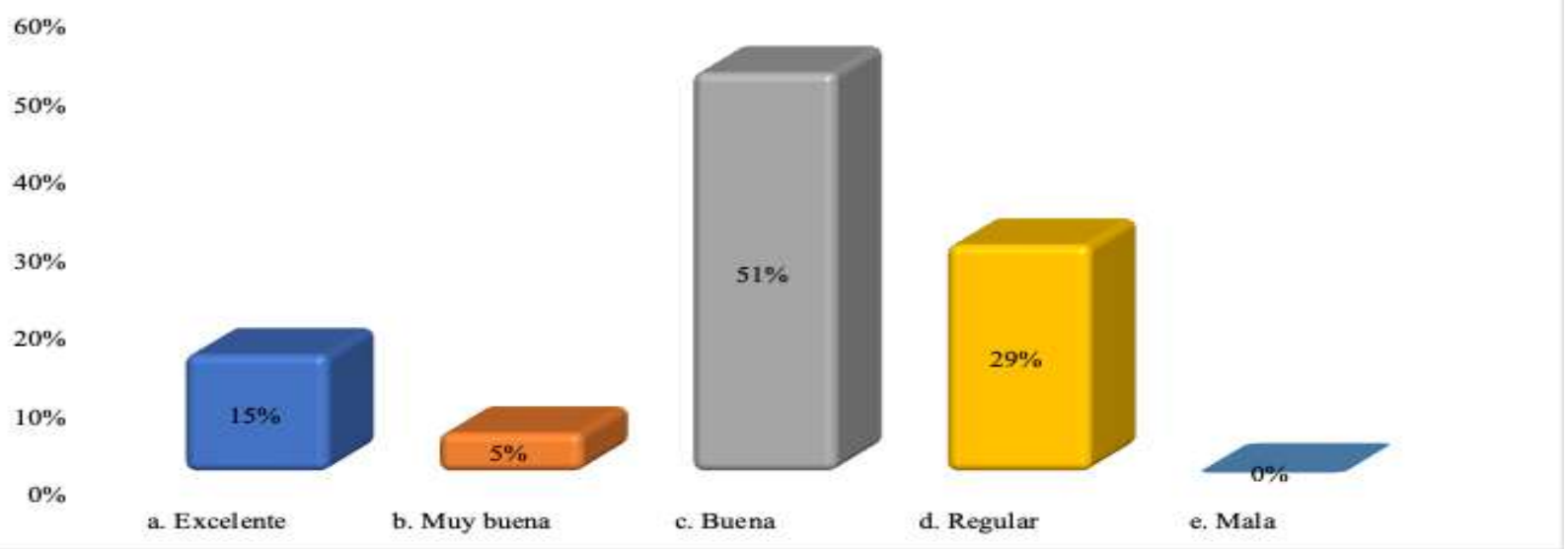

Fuente: Elaboración propia en base a resultados de la investigación.

La imagen 6, muestra que para 51\% de los docentes la interacción con los estudiantes, colegas y directivos de la institución ha sido buena, para el 29\% ha sido regular, para el 15\% ha sido excelente y para el $5 \%$ muy buena. 
Imagen 7. Estrategias pedagógicas utilizadas

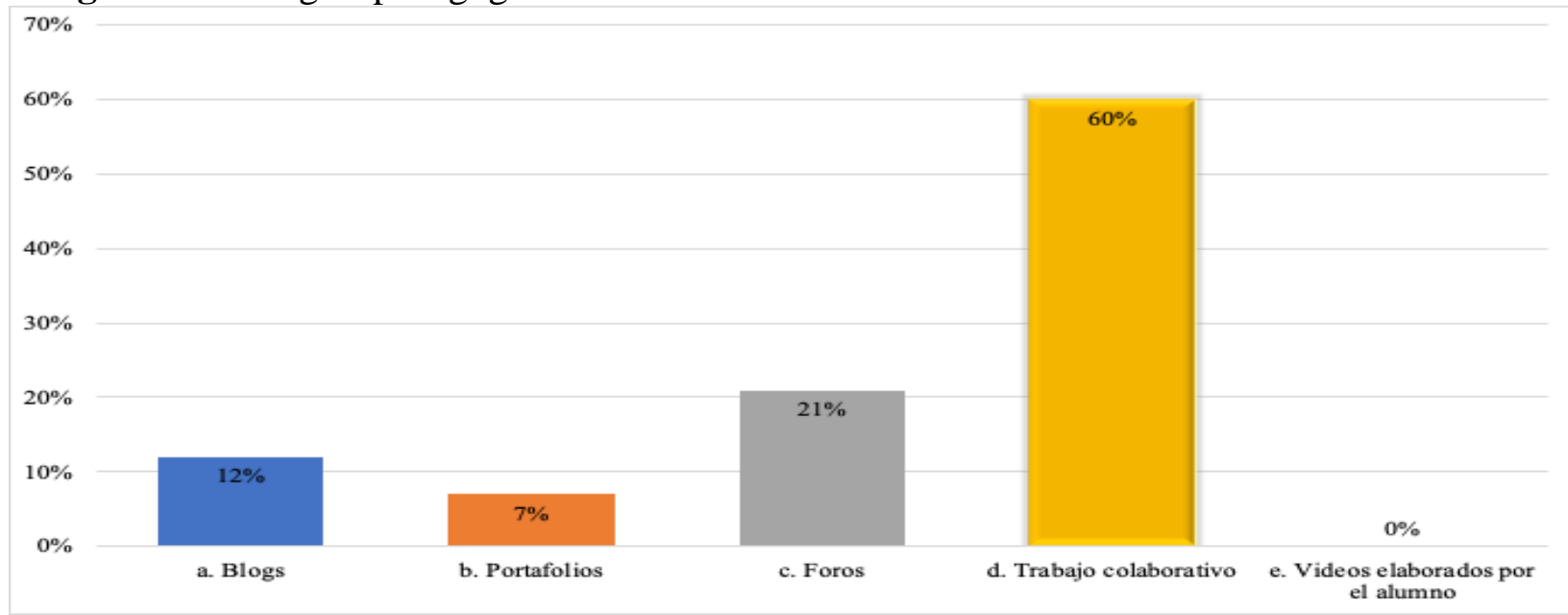

Fuente: Elaboración propia en base a resultados de la investigación.

La Imagen 7, muestra que el 60\% de los docentes utiliza el trabajo colaborativo como estrategia pedagógica para soportar los procesos de enseñanza - aprendizaje, el $21 \%$ los foros, el $12 \%$ los blogs y el 7\% los portafolios. Los resultados anteriores muestran que el docente hace mas uso de trabajo colaborativo, posiblemente lo anterior es consecuencia de la falta de conocimiento del uso de los espacios virtuales.

La Imagen 8, muestra las percepciones de los docentes acerca de los tipos de problemáticas a las que se está enfrentando para transformar sus actividades en cuatro de las principales funciones que realiza dentro del proceso de enseñanza-aprendizaje durante la modalidad a distancia y se describen a continuación:

1. Herramientas de comunicación. Se refiere a los espacios virtuales que se utiliza para poder soportar los procesos de enseñanza-aprendizaje.

2. Contenidos y materiales. Hace referencia a la documentación que se va a enviar/recibir y que va a permitir apoyar los procesos de enseñanza-aprendizaje, así como al formato de estos: documentos, vídeos, presentaciones, etc

3. Seguimiento y control. Se debe contar con un plan que permita especificar la forma de dar retroalimentación acorde al trabajo que están realizando los estudiantes.

4. Mecanismos de evaluación. Cuenta con mecanismos apropiados, que permitan tener certeza de la veracidad de las respuestas por parte de los estudiantes. 
Año 13.

Académica sin Frontera

Núm. 34 Núm. Especial. Tema COVID 19.

ISSN: 2007-8870

https://revistainvestigacionacademicasinfrontera.unison.mx/index.php/RDIASF

Recibido el 20 de agosto de 2020. Dictaminado mediante arbitraje favorablemente 7 de diciembre de 2020.

Imagen 8. Reto principal del proceso-aprendizaje

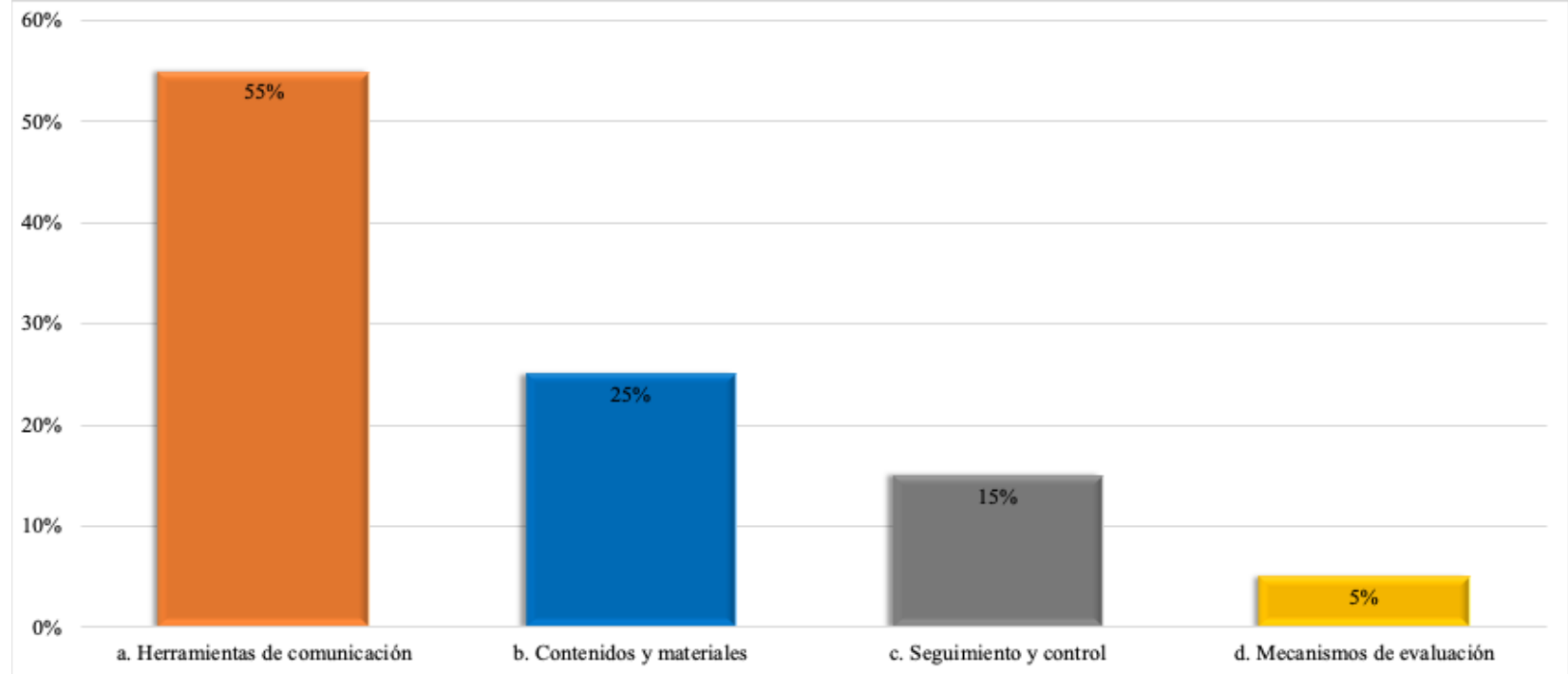

Fuente: Elaboración propia en base a resultados de la investigación.

Los resultados de la misma Imagen 8, muestran que el 55\% considera como un reto principal del proceso de enseñanza-aprendizaje enfrentado por los docentes durante el proceso de educacion a distancia son los espacios virtuales, los resultados muestran que se requiere un espacio de trabajo compartido que permita que la labor docente sea realizada de forma eficiente, contar con recursos tecnológicos adecuados y una capacitación constante alumno y docente que ayude a contar con el uso adecuado de los espacios virtuales para una correcta interacción alumno-docente. Para el $25 \%$ su reto son los contenidos y materiales utilizados. Los resultados muestran la preocupación del docente por el uso de contenidos y materiales con el uso de las TIC's considerando las nuevas condiciones del proceso de enseñanza-aprendizaje mediante espacios virtuales. 


\section{Discusión de resultados}

Los resultados hacen ver que los docente hace más uso de trabajo colaborativo y no hacen uso de las TIC's de forma regular en el proceso de enseñanza - aprendizaje, antes de la pandemia. Asimismo, no utilizan plataformas virtuales como medio de aprendizaje, por lo que el uso de Email, es el medio más común de comunicación cuando quiere realizar contacto con el alumno durante el confinamiento. Desde la perspectiva del docente, existe una preocupación por adaptarse a las nuevas condiciones considetando su problemática en el uso y manejo de los espacios virtuales y la implementación de modelos de educación virtual, lo que hace suponer que el docente está consciente de la importancia que tiene en su trabajo las TIC's como estrategias metodológicas del proceso de enseñanza-aprendizaje. Para el docente, es necesaria la comunicación y el acercamiento con personas de su entorno común, lo cual permite ver la necesidad de afecto del profesor. A pesar de las funciones relacionadas con seguimiento, control y mecanismos de evaluación son importantes en el desarrollo del proceso de enseñanza aprendizaje, para el docente no tenian un grado de importancia alta, pero en muchos casos no les resulta fácil poder hacer un seguimiento de las actividades realizadas.

\section{Conclusiones}

Si bien es cierto, la situación de confinamiento derivada de la emergencia sanitaria ha supuesto la adaptación urgente de la docencia presencial, de forma improvisada, y sin contar con los recursos necesarios para una correcta transformación digital a una docencia a la modalidad virtual. Sin embargo, dentro de las Instituciones de Educación Superior por su naturaleza, debe considerarse el proceso de enseñanza - aprendizaje como una actividad constante y debe ser suficiente, pertinente y estar muy bien diseñada para apoyar realmente el aprendizaje del alumno.

El coronavirus ha venido a cambiar de forma instantánea la forma en que se imparte la educación, ya que la escuela y el hogar, ahora se convierten en el mismo lugar tras las necesarias regulaciones efectuadas. Los resultados de la investigación que aquí se presentan, permite dar validez al objetivo planteado al identificar los principales retos que desde la perspectiva de los docentes, enfrentan en la aplicación del proceso de enseñanza - aprendizaje durante la contingencia causada por el COVID-19. Lo anterior permite al docente universitario, contar con 
Año 13.

Núm. 34 Núm. Especial. Tema COVID 19.

ISSN: 2007-8870

https://revistainvestigacionacademicasinfrontera.unison.mx/index.php/RDIASF

Recibido el 20 de agosto de 2020. Dictaminado mediante arbitraje favorablemente 7 de diciembre de 2020.

información que ayude a mejorar los procesos de enseñanza, de investigación y trabajar en pedagogías alternativas como parte de los principales retos ante la nueva normalidad de la educación.

Lo anterior pone de manifiesto la viabilidad del presente proyecto de investigación, definiendo con los resultados aquí presentados, que la pandemia del COVID-19 independiente de la gravedad de la situación de la crisis a nivel mundial, saca a relucir ciertos aspectos del ámbito educativo en la cual las Instituciones de Educación Superior como la Universidad de Sonora están muy distantes. Si bien en el mundo el uso del smartphone es cosa corriente, el manejo de la tecnología y la transmisión de conocimiento a través de la tecnología es otra cosa totalmente distinta. Están saltando a la luz aspectos importantes a mejorar, una vez superada la guerra contra el virus en la cual está inserta hoy día la población.

A modo de conclusión final, es importante que el docente universitario se de a la tarea de hacer una reflexion de su desempeño, y que vea la situación vivida como un reto que le permita replantear sus estrategias para la mejora del proceso de enseñanza - aprendizaje, buscar formarse y desarrollarse bajo el contexto de la nueva modalidad enfocado en el desarrollo pedagogico y didáctico para la enseñanza y el aprendizaje de los universitarios con el fin de incrementar sus competencias y habilidades en tecnologia para el aprendizaje y el conocimiento que ayude a ofrecer un servicio de calidad a sus alumnos.

Por otro lado, la principal recomendación en este momento de pandemia y de aislamiento, el docente sólo debe abocarse a desarrollar evaluaciones de proceso, tendientes a lograr un seguimiento del aprendizaje del alumno, y no se avance hacia evaluaciones conducentes a calificar y acreditar saberes. Este estudio presenta varias limitaciones que sugieren sean trabajos en vías de investigación futuras. La percepción tambien debe medirse desde la perspectiva del 
alumno, es un resultado que debe analizarse para descartar la relatividad de las dos partes que aplican el proceso de enseñanza - aprendizaje.

\section{Referencias}

Álava Mieles, I.A., Illescas Zaruma, M.S. y Loor Delgado, E. A. (2017). La Plataforma Virtual como fortalecimiento al proceso enseñanza-aprendizaje en las aulas de la Educación Ecuatoriana. Revista Científica de Ciencia y Tecnología, 17(16), 13-24.

Alcívar Trejo, C., Vargas Párraga, V., Calderón Cisneros, J., Triviño Ibarra, C., Santillan Indacochea, S., Soria Vera, R. y Cardenas Zuma, L. (2019). El uso de las TIC en el proceso de enseñanza- aprendizaje de los docentes en las Universidades del Ecuador. Revista ESPACIOS, 40(2), 27-39.

Archer Svenson, N. y De Gracia, G. (2020). Educación superior y COVID-19 en la República de Panamá. ESAL - Revista de Educación Superior en América Latina. 8, 1-5.

Barráez, D. P. (2020). La educación a distancia en los procesos educativos: Contribuye significativamente al aprendizaje. Revista Tecnológica-Educativa Docentes 2.0, 8(1), 4149.

Barrios, F. (2013). Análisis de las megatendencias de Educación Superior. Apuntes Ciencias Sociales; 3(2):185-196.

García-García, M. (2020). La docencia desde el hogar. Una alternativa necesaria en tiempos del Covid 19. Polo del Conocimiento, 304-324. doi:http://dx.doi.org/10.23857/pc.v5i4.1386

González-Díaz, R. R., y Perez, L. A. B. (2015). Análisis financiero empresarial del sector comercio como factor de competitividad través de la lógica difusa. Estrategia, 1(1), 110.

González-Díaz, R. R., Lara, R. J. V., López, R. O. y Hernández-Royett, J. (2016). Tax on advertising and commercial advertising: An analysis from Municipal Tax Management. Globalciencia, 2(1), 20-34.

Granda Asencio, L. Y., Espinoza Freire, E. E., y Mayon Espinoza, S. E. (2019). Las TICs como herramientas didácticas del proceso de enseñanza-aprendizaje. Conrado, 15(66), 104-110. 
Año 13.

Núm. 34 Núm. Especial. Tema COVID 19.

ISSN: 2007-8870

https://revistainvestigacionacademicasinfrontera.unison.mx/index.php/RDIASF

Recibido el 20 de agosto de 2020. Dictaminado mediante arbitraje favorablemente 7 de diciembre de 2020.

Hodges, C., Moore, S., Lockee, B., Trust, T. y Bond, A. (2020). The difference between emergency remote teaching and online learning. Educause Review. Recuperado de https://bit.ly/3b0Nzx7

Lorca, A. A., Cuenca, J. M., Vázquez-Bernal, B., \& Lorca, J. A. (2016). ¿Qué concepciones tienen los docentes en ejercicio y en formación inicial, sobre el uso didáctico de los videojuegos? En J. L. Bravo Galán (Ed.) 27 Encuentros de Didáctica de las Ciencias Experimentales; 543-551, Badajoz: UEX-APICE. ISBN: 978-84-617-4059-8.

Navarro-Ibarra, L., Robles-Aguilar, A., Leyva, J. C. A., y Lugo, F. de J. C. (2016). Secuencia didáctica apoyada en tecnología para la construcción del concepto derivada en problemas de optimización. UNIÓN, Revista Iberoamericana de Educación Matemática, (46), 171187.

Suárez Monzón, N. (2020). Formación docente universitaria y crisis sanitaria COVIS-19. CienciAmérica, Vol. 9 (2), 109 - 114.

Villafuerte, J., Cevallos, Y. P., y Vidal, J. O. B. (2020). Rol de los docentes ante la crisis del COVID-19. Una mirada desde el enfoque humano. REFCalE: Revista Electrónica Formación y Calidad Educativa. ISSN 1390-9010, 8 (1), 134-150. 


\section{Directorio Institucional}

\section{Dr. Enrique Fernando Velázquez Contreras}

Rector

Dr. Ramón Enrique Robles Zepeda

Secretario General Académico

Dra. Rosa María Montesinos Cisneros

Secretaria General Administrativa

Dr. Rodolfo Basurto Álvarez

Director de Vinculación y Difusión

Dra. Adriana Leticia Navarro Verdugo

Vicerrectora de la Unidad Regional Sur

Dr. Ernesto Clark Valenzuela

Director de la División de Ciencias Económicas y Sociales

Dr. Francisco Espinoza Morales

Secretario de la División de Ciencias Económico y Sociales

Dra. Leticia María González Velásquez

Jefe del Departamento de Ciencias Económico Administrativas

Dra. Lidia Amalia Zallas Esquer

Jefe de Departamento de Ciencias Sociales 


\title{
Año 13.
}

Núm. 34 Núm. Especial. Tema COVID 19.

ISSN: 2007-8870

https://revistainvestigacionacademicasinfrontera.unison.mx/index.php/RDIASF

Recibido el 20 de agosto de 2020. Dictaminado mediante arbitraje favorablemente 7 de diciembre de 2020.

\section{Comité Directivo}

\author{
Editor Responsable \\ Dr. Francisco Espinoza Morales \\ Universidad de Sonora \\ Directora \\ Dra. Leticia María González Velásquez \\ Universidad de Sonora \\ Subdirector \\ Dr. Javier Carreón Guillen \\ Universidad Nacional Autónoma de México \\ Editor Científico \\ Dr. Cruz García Lirios \\ Universidad Autónoma del estado de México \\ Master Gráfico \\ M.T.I. Francisco Alan Espinoza Zallas \\ Universidad Estatal de Sonora
}

Nos complace anunciar que su diario, "Academic Research Journal Withoutborders" (ISSN/EISSN 20078870) fue evaluado positivamente en la indexación Citefactor, ahora la página de la revista está disponible en línea, en caso de cualquier problema.

Journals Master | International Innovative Journal Impact Factor (IIJIF)

Red Latinoamericana de revistas Académicas en Ciencias Sociales y Humanidades

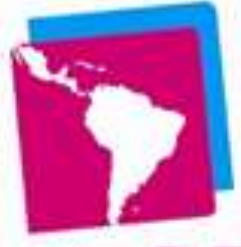




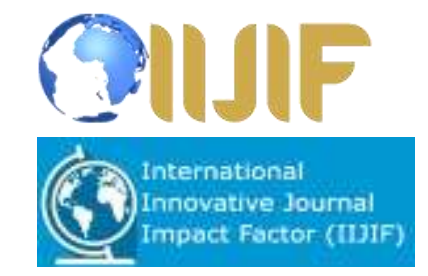

\section{Comité editorial}

Dra. Angélica María Rascón Larios

Universidad de Sonora. México

Dra. María del Rosario Molina González

Universidad de Sonora

Dra. Francisca Elena Rochin Wong

Universidad de Sonora. México

Dra. Lidia Amalia Zallas Esquer

Universidad de Sonora. México

Dra. Beatriz Llamas Arechiga

Universidad de Sonora. México

Dr. Rogelio Barba Álvarez

Universidad de Guadalajara. México

Dra. Rosa María Rincón Ornelas

Universidad de Sonora. México

Dr. Juan Flores Preciado

Universidad de Colima. México

Dr. Amado Olivares Leal. Universidad de Sonora

Universidad de Sonora. México

Dr. Guillermo Velázquez Valadez.

Instituto Politécnico Nacional (IPN) México

Dr. Hugo Nefstalí Padilla Torres.

Universidad Estatal de Sonora. México

Dr. Luis Ramón Moreno Moreno.

Universidad Autónoma de Baja California. México

Dr. Miguel Ángel Vázquez Ruiz.

Universidad de Sonora. México

Dra. Lorena Vélez García.

Universidad Autónoma de Baja California. México 
Año 13.

Núm. 34 Núm. Especial. Tema COVID 19.

ISSN: 2007-8870

https://revistainvestigacionacademicasinfrontera.unison.mx/index.php/RDIASF

Recibido el 20 de agosto de 2020. Dictaminado mediante arbitraje favorablemente 7 de diciembre de 2020.

Dra. Pabla Peralta Miranda.

Universidad Simón Bolívar, Barranquilla, Colombia

Mtro. Roberto Espíritu Olmos

Universidad de Colima (FCA Tecomán) Colima

Dr. Héctor Priego Huertas.

Universidad de Colima (FCA Tecomán) Colima

Mtra. María Guadalupe Alvarado Ibarra.

Universidad de Sonora. México.

MSc. Celso Germán Sánchez Zayas

Universidad de Camagüey, Ignacio Agramonte Loynaz, Cuba

Dra. María Luisa Quintero Soto

Universidad Autónoma del Estado de México

Dr. Eyder Bolivar Mojica

Universidad Católica, Luis Amigó, Medellin, Colombia

Revisores de Textos en Inglés

Mtro. Renato Encinas

Mtra. Cecilia Guadalupe Martínez Solano

\section{Comité científico}

Dr. Rosendo Martínez Jiménez. Universidad Autónoma Benito Juárez de Oaxaca.

Dr. Hugo Neftalí Padilla. Universidad Estatal de Sonora

Dra. María Teresa Gaxiola Sánchez. Universidad de Sonora.

Dr. José Cesar Kaplan. Universidad Estatal de Sonora.

Dr. Alfredo Islas Rodríguez. Universidad de Sonora

Frecuencia de publicación: semestral / 2 números por año y en ocasiones edición especial. 
Revista de Investigación Académica sin Frontera (RIASF) con (ISSN: 2007-8870) es un interlocutor internacional de acceso abierto revisado diario en línea en el ámbito del de las Ciencias Económicas Administrativas y Sociales. Su objetivo principal es dar a los trabajos de investigación de calidad. Cubre todas las sub-campos de los campos anteriormente mencionados. Proporciona la plataforma a académicos, estudiantes y profesionales. Sólo pública trabajos de investigación y artículos de revisión inicial. Documento presentado debe cumplir con algunos criterios como, debe ser original, inédita y no estén sometidos a ninguna otra revista.

RIASF es una revista arbitrada / Revisión por pares International. Publicamos documentos sobre una variedad de temas, contextos y estrategias de análisis que examinan la relación entre la rápida evolución para la Sociedad y la tecnología del conocimiento.

REVISTA DE INVESTIGACIÓN ACADÉMICA SIN FRONTERA, Año 13, No. 34 Especial, tema COVID 19, Julio - diciembre 2020, es una publicación semestral de investigación científica, editada por la Universidad de Sonora, a través de las División de Ciencias Económicas y Sociales, de la Unidad Regional Sur, Blvd. Lázaro Cárdenas No. 100, Col. Francisco Villa, Navojoa, Sonora, Sonora, México, C.P. 85880. Tel. (642) 42599-54.

https://revistainvestigacionacademicasinfrontera.unison.mx/index.php/RDIASF

I, revistaacademicasinfrontera@unison.mx.

Editor responsable: Francisco Espinoza Morales. Reserva de Derechos al Uso Exclusivo: 04-2013121811323700-203 e ISSN: 2007-8870, ambos otorgados por el Instituto Nacional de Derecho de Autor. Inscrita en el Directorio de LATINDEX, con Núm. De folio 20014, folio único 14590. Responsable de la última actualización de este Número, Unidad Informática de la Universidad de Sonora, fecha de la última modificación, 30 de diciembre 2020, indexada a Cite Factor Academic Scientific Journal y Journals Master (IIJIF) y Red Latinoamericana de Revistas Académicas en Ciencias Sociales y Humanidades, (Latín Rev). Las opiniones expresadas por los autores no necesariamente reflejan la postura del editor de la publicación. Se autoriza la reproducción total o parcial de los contenidos e imágenes en la presente publicación siempre y cuando se cuente con la autorización del editor y se cite plenamente la fuente. 


\section{Año 13.}

Revista de Investigación

Núm. 34 Núm. Especial. Tema COVID 19.

Académica sin Frontera

ISSN: 2007-8870

https://revistainvestigacionacademicasinfrontera.unison.mx/index.php/RDIASF

Recibido el 20 de agosto de 2020. Dictaminado mediante arbitraje favorablemente 7 de diciembre de 2020.

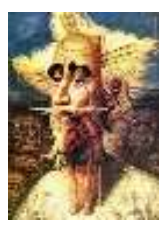

Nos complace anunciar que su diario, "Academic Research Journal Withoutborders" (ISSN/EISSN 20078870) fue evaluado positivamente en la indexación Citefactor, ahora la página de la revista está disponible en línea, en caso de cualquier problema.

Journals Master | International Innovative Journal Impact Factor (IIJIF)

\section{Red Latinoamericana de revistas Académicas en Ciencias Sociales y Humanidades}
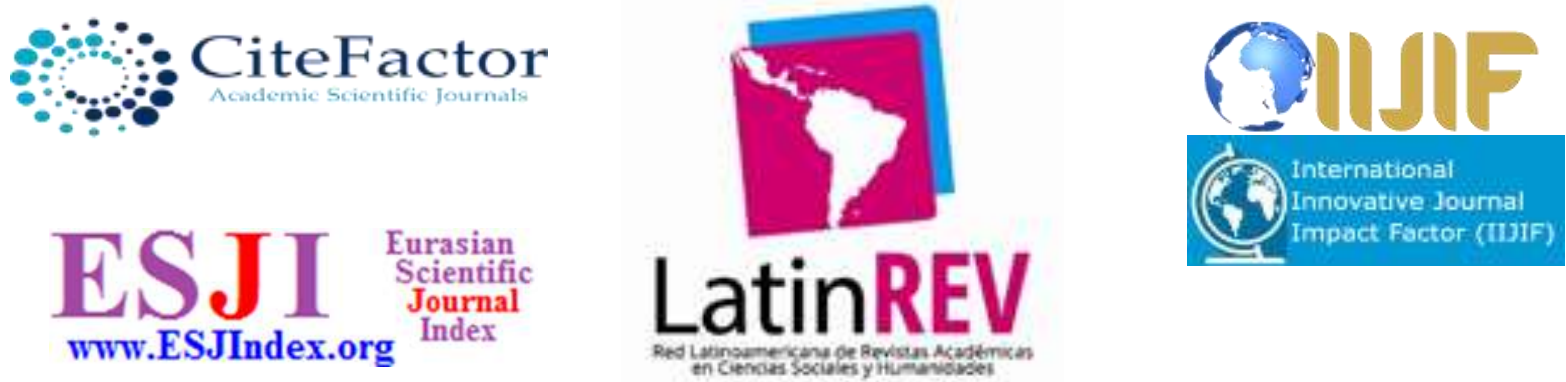

\section{https://www.neliti.com}
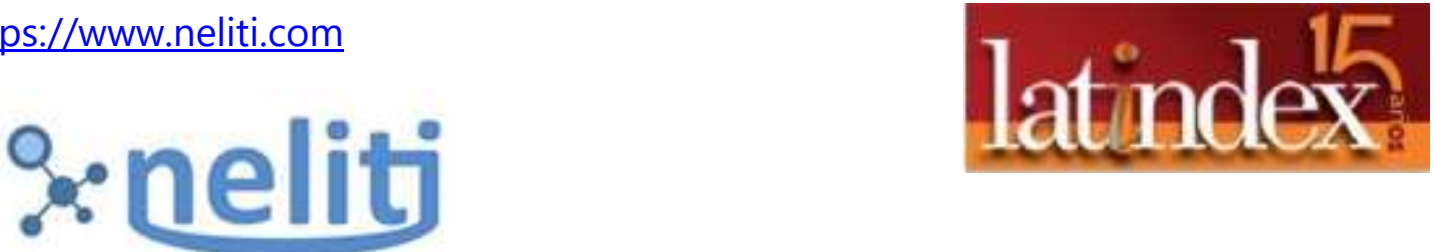

Indonesia's Research Repository

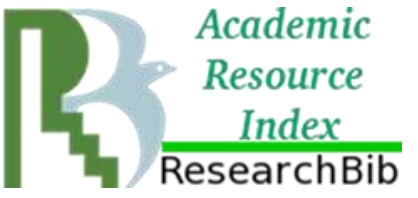


